\title{
Research on Routing Mechanism of Wireless Sensor Network Based on Cooperative Transmission
}

\author{
TIAN Ye-fei \\ ChongQing college of electronic engineering, Chongqing, China \\ Emai:183348163@qq.com
}

Keywords: Routing Mechanism, Wireless Sensor Network, Cooperative Transmission

\begin{abstract}
This paper presents a wireless sensor network cooperative transmission protocol, analyzes its transmission efficiency and improvement of network throughput, and then obtains the scope of the cooperative transmission protocol. The cooperative transmission protocol is particularly suitable for wireless environments with reliable and high-speed data transmission requirements and poor channel environment. Proper use of this protocol can improve the transmission efficiency of sensor nodes, save network energy and improve network.
\end{abstract}

\section{Introduction}

In the wireless sensor networks (WSN), the energy of the sensor nodes is often strictly limited, and wireless communication is the most energy-consuming operation. In harsh wireless environments, channel fading, inter-channel interference, and irregularity of radio waves present a challenge for wireless communications to accelerate the energy consumption of sensor nodes. In this case, the importance of transmission technology is highlighted because it reduces the impact of fading and is an effective anti-fading measure with the advantage of improving system performance without increasing transmits power or bandwidth. Multi-input multiplexing (MIMO) technology uses multi-antenna spatial transmission to suppress channel fading, and the channel capacity increases linearly as the number of antennas increases. That is, it is possible to increase the radio channel capacity doubly using the MIMO channel, and the spectrum utilization rate can be multiplied without increasing the bandwidth and the antenna transmission power. However, MIMO technology cannot be directly applied to WSN, because in most cases, nodes in WSN not only the energy is limited, its volume is limited, but also consider its cost cannot be too high, which resulted in the It is not a practical way to install multiple antennas on a common WSN node. In view of this situation, the cooperative transmission mechanism has been raised, its characteristics are mainly reflected in two aspects: (1) by a group of single antenna equipment to imitate the antenna array. (2) high-capacity, high-reliability communication systems with low-cost single devices. Because its function is similar to that of MIMO, some of the literature is also called virtual-MIMO or cooperative MIMO. Collaborative transmission has a broad application prospect in WSN because the sink in the WSN is a center of data collection and processing, and in many cases it is close to the rear and can continue to be powered by its energy consumption, volume, etc. The limitations cannot be considered in relation to ordinary sensor nodes, so it can be complicated. Thus in the WSN, a single sink node can be used to complete the function of receiving, and by a number of volume and energy constraints of the common sensor nodes to complete the function of sending.

\section{The Cooperative Transmission Protocol}

Although the cooperative transmission is not necessarily to be established on the clustering protocol, it is generally necessary to determine the set of nodes used for cooperative transmission or (and) reception in the cooperative transmission. The cooperative transmission based on the clustering protocol has its natural advantages. Thus, this paper presents a single-hop cooperative transmission based on clustering (SCTC) based on clustering for WSN. Suppose the receiver, the sink node, has no energy and volume constraints, it is equipped with an antenna array because the actual application of the sink node can be able to move the car or reconnaissance aircraft. At the same time assume that the system is synchronized, all nodes to the sink are hop, and the node's transmit power 
is adjustable.

The protocol can be divided into four steps: the first step, the cluster stage and the cluster head $(\mathrm{CH})$, the cluster, and then determine the cooperative nodes (cooperative nodes) in each cluster. Two steps, the information transmission within the cluster, that is, $\mathrm{CH}$ to the cluster $\mathrm{CNs}$ to send control information and data; the third step, the cluster CNs together to the sink node for data transmission; the fourth step, the sink node to receive and detect the signal.

\section{Introduction and Research Significance of Wireless Sensor Networks}

As a result of sensor technology, low-power electronics and radio frequency technology advances, low-power, inexpensive wireless sensor to large-scale application, the corresponding wireless sensor network (Wireless Sensor Network, WSN) came into being. The network is composed of self-organizing network, which is composed of sensor, data processing unit and communication module. It is called the sensor network. If the communication medium between nodes is wireless, then it is called wireless sensor network. The wireless sensor network integrates sensor technology, distributed information processing technology, embedded computing technology and wireless communication network technology, can cooperate in real-time monitoring, perception and collection of various environmental or monitoring object information and processing, to the needs of users. Compared with traditional center processing, it has the advantages of high accuracy, high robustness, high flexibility and strong intelligence. It can be applied to national defense, national security, environmental monitoring, traffic management, manufacturing, medical and health, anti-terrorism disaster and other areas.

Due to the huge application prospects of wireless sensor networks, in 1999 and 2003, "Business Week" and MIT "Technical Review" in the forecast of future technology development report, respectively, wireless sensor technology as the 21st century, the most influential 21 technology And one of the 10 new technologies to change the world. The tremendous value of the sensor network has attracted the attention of the military, academia and industry in many countries of the world. The Natural Science Foundation of the United States established a sensor network research program in 2003, investing \$ 34 million to support the relevant underlying theoretical research. The US Department of Defense and the military departments have given a high degree of attention to the sensor network, the establishment of a series of military sensor network research projects, such as the famous US Department of Defense (DARPA) project. The United States MIT, Berkley, Cornell, University of Southern California and MICROSOFT, INTEL, CROSSBOW, HP, Omron and other international companies have invested a lot of human, material and financial resources for research and development of sensor network technology, standards and products of the higher schools and research institutions have also carried out some effective research. But on the whole, these studies are still in the initial stage, from the practical application is also very different.

\section{The Characteristics of Wireless Sensor Networks}

Wireless sensor networks, as a special wireless ad hoc network, are different from traditional wireless networks and present new challenges and research questions. (1) The purpose is to collect and process the data from the environment in a timely and reliable manner, passing the data to the sink node (Sink), which is data-centric and its communication mode has many to many features The new communication model poses new challenges to the protocols designed for the many-to-many, one-to-many communication modes. In addition, the multi-hop transmission is also characteristic because the sink node is far from the source node. (2) due to cost considerations, the node must be subject to cost constraints, so its energy, CPU computing speed, memory and communication bandwidth and other resources must be greatly constrained, especially because it is difficult to consider charging problems, energy has become a very Important resources. (3) wireless sensor network with a large number of nodes, large-scale, large-scale features. This allows the protocol design to take into account the need for harmonization of the entire network. (4) There are many unpredictable factors in the wireless sensor network, which are mainly due to the unpredictability 
caused by the highly dynamic environment, the unpredictability caused by the vulnerability of the nodes and the unpredictability of the communication capacity caused by the dynamic changes of the network communication members. This allows the protocol to have the ability to adapt to unpredictable factors. (5) The purpose of the sensor network is to collect useful information from the environment, rather than simply transfer the data, so the network information processing becomes very important, is different from the general wireless self-organizing network and sensor array of the main features. (6) sensor networks Many applications have different levels of real-time transmission and reliability requirements, such as in video surveillance applications, the delay and packet loss problems on the monitoring results is obvious, such as in the battlefield data acquisition application requires data acquisition is very timely and reliable.

\section{The Wireless Sensor Network System Based on Collaborative Transmission}

Node Hardware Device Improvement. The use of cooperative transmission technology will lead to the improvement of the hardware complexity of the sensor node. As mentioned earlier, the end node needs to estimate the instantaneous signal-to-noise ratio of the channel and, after receiving the source and relay transmission, two independent fading channels of the signal after the need for complex signal detection to determine the signal, the node of the hardware devices are made a very high demand. The sensor node is a miniature embedded device and the price of low power consumption is small, which requires the improvement of the sensor node hardware equipment, so that it has a certain processing power and storage capacity. The

Sync Problems. In this paper, it is assumed that the system has good synchronization, because the synchronization between terminals is to ensure that co-transmission can be effective and necessary conditions. For infinite sensor networks, synchronization between nodes is difficult to achieve. In the sensor network, the cost of the nodes cannot be too high, the small size of the nodes cannot be installed in addition to the local oscillator and wireless communication module for more synchronization devices, and the sensor network with a limited number of nodes with limited energy, Will consume energy. Can be used in the wireless sensor network to overcome the synchronization mechanism to synchronize the problem, the sender sends the signal first stored in the cache, when fully received the signal from the relay, the destination and then remove the signal from the cache, the two Signal samples to detect and judge. The

Collaborative Transmission in the Case of Network Dynamics. In this paper, the system model assumes that the position of each sensor node and sink is fixed, but the topology of the sensor network may change due to factors such as environmental factors or power consumption caused by sensor nodes failure or failure. In the case of changing the network topology, you can use the protocol in the use of collaborative transmission of the system allows the system to better adapt to this change. For example, in the case of relay failure, the receiver according to the channel quality is good or bad to inform the sender to find another relay in a timely manner: the receiver fails, the relay can directly replace the node as a routing node without having to re-establish Routing, which can effectively increase the robustness of the network, and save the energy needed to rebuild the route to extend the network lifetime.

From the different network or the interests of different operators, heterogeneous network interconnection structure model can be divided into essentially: integrated mode or peer-to-peer model. Integrated mode from an infrastructure and backbone network, other terminals or local area network through the information infrastructure and backbone network access to future wireless networks, thus access to the future wireless network "any time, any place, between any more Kind of information transmission "service. This method is efficient, mature technology, suitable for infrastructure or backbone network of traditional operators to achieve internal network interconnection or integration of other networks, so the traditional operators and backbone network operators to actively promote the way, the current UTMS and Most of the literature on WLAN network interconnection is studied on the basis of this structural model. In this mode, each access network in accordance with the infrastructure and backbone requirements of the interface mode access to infrastructure and backbone network, in accordance with the infrastructure and backbone 
network control and management to coordinate the work in order to achieve the purpose of collaborative work. Peer-to-peer mode refers to the interconnection between heterogeneous networks using peer-to-peer networks. All terminals and access networks try to provide peer-to-peer services on peer-to-peer management control principles. This approach in line with the network of personalized, diversified service needs and development direction is the emerging technology and operators hope. As the form of peer-to-peer network is more able to reflect the nature of the network interconnection network characteristics, it is the future heterogeneous wireless network collaborative work system cannot be ignored as a structural model. However, due to the interconnection of interconnection nodes requirements for interconnection nodes, there are still some theoretical, technical and protocol issues that need to be studied and solved in practice.

\section{Conclusion}

The cooperative transmission mechanism in WSN is a relatively new research field. It draws on the idea of MIMO. Many existing MIMO research results, such as coding algorithm, channel estimation and channel equalization, can be used for reference. At present, domestic and foreign research in this area has just started, there are still many problems, for example, most of the current research is based on WSN nodes have been synchronized on the basis of the. Although the effect of S. Jagannathan has not had a significant effect on co-transmission when the time synchronization error is within $10 \%$, and some studies have begun to study cooperative transmission mechanisms that do not require accurate clock synchronization, but the synchronization between cooperative nodes is still a prominent problem in collaborative application of WSN. In addition, trade-offs such as increased load due to cooperative transmission and performance improvements brought about by it, and the application of the fading channel model is the challenge of collaborative delivery in WSN applications. However, it is the co-transmission mechanism proposed to promote the practical use of MIMO technology. Moreover, this technology can be used for wireless self-organizing network, wireless LAN and wireless sensor networks and other occasions, has a broad application prospects.

\section{Acknowledgment}

This work is supported by Scientific and Technological Research Program of Chongqing Municipal Education Commission(Grant No. KJ1729404).

\section{References}

[1] Huifang Zhou: Journal of Information, Vol. 6 (2004) No 53, p.25-26

[2] Hongli Zhang: Computer Education, Vol. 12 (2005) No 27, p.74-76

[3] Qin Guo: Computer and Network, Vol. 1 (2006) No 33, p.11-14

[4] Jieming Liu: Guangxi Normal University, Vol. 3 (2007) No33, p.121-124

[5] Jia Xinzhang, Li Jingyuan. Electronic Industry, Vol. 6 (2014) No 53, p.25-26 